\title{
Epigenetic regulation of enhancer RNAs in neuropsychiatric disease and addiction
}

\author{
Evan J Kyzar ${ }^{1,2}$ (iD) \& Subhash C Pandey*,1,2,3 (iD \\ ${ }^{1}$ Center for Alcohol Research in Epigenetics, Department of Psychiatry, University of Illinois at Chicago, Chicago, IL 60612, USA \\ ${ }^{2}$ Jesse Brown Veterans Affairs Medical Center, Chicago, IL 60612, USA \\ ${ }^{3}$ Department of Anatomy \& Cell Biology, University of Illinois at Chicago, Chicago, IL 60612, USA \\ *Author for correspondence: Tel.: 312413 1310; Fax: 312996 7658; scpandey@uic.edu
}

\begin{abstract}
“'Enhancer sites are crucial regulators of gene expression in development and in adult tissue, coordinating cell-type specificity and transcriptional programs. Many of these eRNAs are transcribed in response to neuronal depolarization, indicating that they might play a role in crucial activity-dependent regulatory activities"
\end{abstract}

First draft submitted: 5 April 2020; Accepted for publication: 16 April 2020; Published online: 13 August 2020

Keywords: addiction • alcohol • enhancer RNA • epigenetics • non-coding RNA

Genomic regulation is extremely complex and is regulated by both intracellular signaling mechanisms and the extracellular environment. Cis-regulatory elements known as enhancers regulate the expression of specific target genes and are crucial for normal development and cellular functions [1]. Interestingly, some enhancers are actively transcribed, producing non-coding RNAs (ncRNA) known as enhancer RNAs (eRNAs) [1,2]. These eRNAs are regulated by epigenetic mechanisms and have been shown to interact with other regulatory proteins including transcriptional machinery at promoters and to promote chromatin looping to refine robust control of gene expression mechanisms $[1,3]$.

In 2010, Kim et al. [2] made the groundbreaking discovery that eRNAs are transcribed bidirectionally from cis-regulatory enhancer regions. Enhancer sites are crucial regulators of gene expression in development and in adult tissue, coordinating cell-type specificity and transcriptional programs [4]. Many of these eRNAs are transcribed in response to neuronal depolarization, indicating that they might play a role in crucial activity-dependent regulatory activities. Further analysis showed that active enhancers enriched in $\mathrm{H} 3 \mathrm{~K} 27 \mathrm{ac}$ are much more likely to be transcribed by RNA Pol II than inactive or poised enhancers enriched in H3K27me3 [2]. Most eRNAs are uncapped and therefore subject to rapid turnover within the nucleus [5]. Additionally, members of the CREB protein family including CBP and p300 appear to coordinate cellular transcriptional responses at eRNA sites $[2,4,6]$.

Early work on eRNA biogenesis and function has revealed that eRNAs are regionally specific within the brain in a manner that likely also extends to cell-type specificity [7]. Interestingly, many eRNAs are located near or within IEGs including $A r c$, Fos (which encodes the cFos protein), Gadd45b and neuronal Npas 4 and are rapidly transcribed in response to neuronal activity [2,8-10]. These genes coordinate the activity-dependent epigenetic changes via their actions as chromatin modifiers and transcription factors, thus facilitating activity-dependent synaptic plasticity and behaviors including learning and complex emotional expression. Notably, eRNA transcription precedes mRNA transcription at activity-dependent IEGs [2,8,9], suggesting that eRNAs may play a role in activating and/or maintaining IEG expression in response to environmental stimuli.

Upon their discovery, many questions arose regarding eRNA function. Among the most pressing was whether eRNAs are functional molecular entities or simply byproducts of promiscuous Pol II binding in response to increased levels of cellular or neuronal activity. Mechanistic studies began to shed light in this direction by showing that some eRNAs interact with the local chromatin environment to facilitate expression of target mRNAs. For example, the $A r c$ eRNA is encoded from a SARE site located approximately 7 kilobases upstream of the $A r c$ mRNA transcription start site and robustly responds to neuronal depolarization via a CREB response element [9]. Arc eRNA transcriptional activity is associated with enhancer-promoter looping, bringing the SARE site in closer proximity to

Future $\because$ Medicine 
the Arc transcription start site. Arc eRNA binds to a nonspecific RNA recognition motif within the NELF protein, which normally serves to inhibit elongation of Pol II [9]. The eRNA-bound NELF undergoes a conformational change, leading to the disinhibition of Pol II and subsequent transcription of Arc mRNA [9]. This work was critical, as it showed that the Arc eRNA indeed has a functional role apart from merely being a byproduct of widespread transcription.

Recent work from the Pandey lab aimed to determine the contribution of the Arc eRNA to the lasting deficits seen in the amygdala of adult rats after adolescent alcohol exposure, representing one of the first explorations of eRNAs in animal models of neuropsychiatric disease [11]. Adolescence is a critical period in the development of addiction and other psychiatric conditions, as gene expression programs in the brain continue to change and inform adolescent-typical behaviors such as binge drinking. Repeated binge alcohol exposure leads to increased risk for alcohol use disorder (AUD) and negative affective states including anxiety disorders in adulthood, and animal models have recapitulated these behavioral phenotypes and repeatedly shown that chromatin architecture in the amygdala is altered by adolescent alcohol exposure [11,12].

This work has demonstrated that adolescent alcohol exposure leads to decreased expression of Arc eRNA in the adult amygdala. The decrease in Arc eRNA transcription is mediated by decreased KDM6B occupancy of the SARE site and subsequent increased H3K27me3 deposition and decreased H3K27ac occupancy [11]. Importantly, it was shown that the NELF disinhibition of Arc mRNA transcription by Arc eRNA originally shown in neuronal culture [9] appears to also be operative in the rat amygdala [11]. Studies in the human postmortem amygdala found that increased $\mathrm{H} 3 \mathrm{~K} 27 \mathrm{me} 3$ and enhancer of EZH2, and decreased H3K27ac at the SARE site of the ARC gene, are associated with decreased $A R C$ mRNA expression in early-onset AUD patients [13]. Additionally, inhibition of $K d m 6 b$ or direct knockdown of the negative strand of Arc eRNA in the central nucleus of amygdala leads to anxiety-like behaviors, thus mimicking the phenotype observed in adult rats previously exposed to adolescent alcohol [11]. This was the first study that successfully modified behavioral phenotypes by direct inhibition of an eRNA transcript in vivo in brain [11].

Aside from Arc eRNA, the eRNAs surrounding the Fos gene are some of the most well-studied, particularly in the brain. The Fos locus contains five distinct eRNAs that are stimulus-specific, meaning that distinct subsets of eRNAs are transcribed in response to neuronal depolarization, BDNF treatment and cAMP induction via forskolin [8]. Similar to $A r c$, the Fos eRNAs show an association between expression levels and enhancer-promoter proximity. Additionally, the stimulus-specificity of Fos eRNAs is at least partially dependent on transcription factors including CREB and NPAS4 [8]. A more recent study showed that bidirectional epigenetic regulation by CRISPR activation (dCas9-p300) or inhibition (dCas9-HDAC8) altered H3K27ac-associated chromatin dynamics at the Fos e2 eRNA locus, also known as Fos enhancer 2. Increased H3K27ac at this enhancer site facilitated Fos mRNA expression and neuronal burst-firing dynamics [10]. Increased activity at Fos e2 eRNA recruits BRD4 to the chromatin leading to Pol II-mediated transcriptional elongation of Fos mRNA [10]. Aside from furthering the knowledge of eRNA-mediated transcriptional mechanisms in neurons, this work involving well-studied IEGs evokes interesting questions regarding the role of eRNAs and their interaction with chromatin dynamics in the brain in both baseline and diseased states.

Recent studies in animal models have confirmed that many of the in vitro findings translate into brain tissue while additionally linking eRNAs to behavior. First, induction of widespread neuronal activity in the hippocampus via kainic acid leads to marked activity-dependent eRNA transcription and alteration of enhancer-promoter looping [14]. Some of these alterations to chromatin architecture, particularly those involving the cFos-containing AP-1 transcription factor, persist long after induction of neuronal activity [14]. Secondly, response to a conditioned stimulus in motor learning tasks activates eRNA transcription and enhancer-promoter interactions in the cerebellar vermis [15]. Conditional CRISPR-mediated knockout of a core cohesin subunit, which serves to anchor enhancerpromoter loops, leads to loss of enhancer-promoter interactions and motor learning deficits [15].

The perturbation of eRNAs in animal models begs the question of whether eRNAs are also involved in addiction and other neuropsychiatric disorders in humans. This is particularly relevant as the integration of genome-wide Hi$\mathrm{C}$ data showing enhancer-promoter interactions with available Genome-Wide Association Studies data improves the predictive capacity for both psychiatric and neurodegenerative disorders [16]. For example, allelic variation at a conserved enhancer of the GAL gene is associated with increased alcohol intake and anxiety in humans, and CRISPR-mediated knockout of the Gal enhancer decreases alcohol consumption and anxiety-like behavior in male mice [17]. Analysis of differential DNA methylation at enhancer sites in the prefrontal cortex of Alzheimer disease patients revealed a hypomethylated enhancer cluster that appears to activate the BACE1 gene [18]. eRNAs 
transcribed in dopaminergic neurons in the human brain show an enrichment of genetic variants involved in schizophrenia, addiction and Parkinson's disease and this study further identified an eRNA on chromosome 17 regulated by Parkinson's disease-associated gene variation [19]. Lastly, a genome-wide study of prefrontal cortex from schizophrenia patients compared with controls revealed 118 differentially expressed eRNAs and allele-specific regulation of eRNA expression by known schizophrenia risk variants [20].

Taken together, these studies demonstrate the importance of eRNAs in brain health and disease. However, the relative youth of these investigations mean that many questions remain to be answered [5]. What percentage of eRNAs are functional entities compared with those that may be transcriptional noise? Do eRNAs physically activate target gene transcription by chromatin looping, transcription factor binding, homotypic attraction, phase separation or some other unknown mechanism? What role does evolutionary selection play in tissue- and brain region-specific eRNA expression and function? Are eRNAs viable treatment targets or prognostic markers in neuropsychiatric diseases including AUD and other addictive disorders? The interactive role of chromatin dynamics with eRNAs $[1,10,11]$ will likely be the subject of intense investigation in the coming years, as eRNA biology and its effects on brain function and behavior remain ripe for discovery.

\section{Financial \& competing interest disclosure}

This work was supported by National Institute on Alcohol Abuse and Alcoholism Grants UO1AA-019971, U24AA-024605 (Neurobiology of Adolescent Drinking in Adulthood [NADIA] project), R01AA-010005, P50AA-022538 (Center for Alcohol Research in Epigenetics) and by the Department of Veterans Affairs (Merit Grant- 101 BX004517 and Senior Research Career Scientist Award) to SC Pandey, as well as a fellowship F30AA-024948 grant to EJ Kyzar. The authors have no other relevant affiliations or financial involvement with any organization or entity with a financial interest in or financial conflict with the subject matter or materials discussed in the manuscript apart from those disclosed.

No writing assistance was utilized in the production of this manuscript.

\section{Open access}

This work is licensed under the Attribution-NonCommercial-NoDerivatives 4.0 Unported License. To view a copy of this license, visit http://creativecommons.org/licenses/by-nc-nd/4.0/

\section{References}

1. Calo E, Wysocka J. Modification of enhancer chromatin: what, how, and why? Mol. Cell 49(5), $825-837$ (2013).

2. Kim TK, Hemberg M, Gray JM et al. Widespread transcription at neuronal activity-regulated enhancers. Nature 465(7295), 182-187 (2010).

3. Carullo NVN, Day JJ. Genomic enhancers in brain health and disease. Genes (Basel) 10(1), pii:E43 (2019).

4. Wu H, Nord AS, Akiyama JA et al. Tissue-specific RNA expression marks distant-acting developmental enhancers. PLoS Genet. 10(9), e1004610 (2014).

5. Andersson R, Sandelin A. Determinants of enhancer and promoter activities of regulatory elements. Nat. Rev. Genet. 21(2), 71-87 (2020).

6. Bose DA, Donahue G, Reinberg D, Shiekhattar R, Bonasio R, Berger SL. RNA binding to CBP stimulates histone acetylation and transcription. Cell 168(1-2), 135-149.e122 (2017).

7. Yao P, Lin P, Gokoolparsadh A, Assareh A, Thang MW, Voineagu I. Coexpression networks identify brain region-specific enhancer RNAs in the human brain. Nat. Neurosci. 18(8), 1168-1174 (2015).

8. Joo JY, Schaukowitch K, Farbiak L, Kilaru G, Kim TK. Stimulus-specific combinatorial functionality of neuronal c-fos enhancers. Nat. Neurosci. 19(1), 75-83 (2016).

9. Schaukowitch K, Joo JY, Liu X, Watts JK, Martinez C, Kim TK. Enhancer RNA facilitates NELF release from immediate early genes. Mol. Cell 56(1), 29-42 (2014).

10. Chen LF, Lin YT, Gallegos DA et al. Enhancer histone acetylation modulates transcriptional bursting dynamics of neuronal activity-inducible genes. Cell Rep. 26(5), 1174-1188.e1175 (2019).

11. Kyzar EJ, Zhang H, Pandey SC. Adolescent alcohol exposure epigenetically suppresses amygdala Arc enhancer RNA expression to confer adult anxiety susceptibility. Biol. Psychiatry 85(11), 904-914 (2019).

12. Pandey SC, Kyzar EJ, Zhang H. Epigenetic basis of the dark side of alcohol addiction. Neuropharmacology 122, 74-84 (2017).

13. Bohnsack JP, Teppen T, Kyzar EJ, Dzitoyeva S, Pandey SC. The lncRNA BDNF-AS is an epigenetic regulator in the human amygdala in early onset alcohol use disorders. Transl. Psychiatry 9(1), 34 (2019). 
14. Fernandez-Albert J, Lipinski M, Lopez-Cascales MT et al. Immediate and deferred epigenomic signatures of in vivo neuronal activation in mouse hippocampus. Nat. Neurosci. 22(10), 1718-1730 (2019).

15. Yamada T, Yang Y, Valnegri P et al. Sensory experience remodels genome architecture in neural circuit to drive motor learning. Nature 569(7758), 708-713 (2019).

16. Sey NYA, Hu B, Mah W et al. A computational tool (H-MAGMA) for improved prediction of brain-disorder risk genes by incorporating brain chromatin interaction profiles. Nat. Neurosci. doi:10.1038/s41593-020-0603-0 (2020) (Epub ahead of print).

17. Mcewan AR, Davidson C, Hay E et al. CRISPR disruption and UK Biobank analysis of a highly conserved polymorphic enhancer suggests a role in male anxiety and ethanol intake. Mol. Psychiatry doi:10.1038/s41380-020-0707-7 (2020) (Epub ahead of print).

18. Li P, Marshall L, Oh G et al. Epigenetic dysregulation of enhancers in neurons is associated with Alzheimer's disease pathology and cognitive symptoms. Nat. Commun. 10(1), 2246 (2019).

19. Dong X, Liao Z, Gritsch $\mathrm{D}$ et al. Enhancers active in dopamine neurons are a primary link between genetic variation and neuropsychiatric disease. Nat. Neurosci. 21(10), 1482-1492 (2018).

20. Hauberg ME, Fullard JF, Zhu L et al. Differential activity of transcribed enhancers in the prefrontal cortex of 537 cases with schizophrenia and controls. Mol. Psychiatry 24(11), 1685-1695 (2019). 